\title{
Posttraumatic Growth Inventory (PTGI): Adaptação e Validade Fatorial no Nordeste Brasileiro
}

\author{
Emerson Diógenes de Medeiros - Universidade Federal do Pianí, Parnaíba, Brasil \\ Ricardo Neves Couto - Universidade Federal da Paraíba, João Pessoa, Brasil \\ Patrícia Nunes da Fonsếca - Universidade Federal da Paraíba, João Pessoa, Brasil \\ Paulo Gregório Nascimento da Silva - Universidade Federal do Vale do São Francisco, Petrolina, Brasil \\ Paloma Cavalcante Bezerra de Medeiros - Universidade Federal do Piani, Parnaíba, Brasil
}

\begin{abstract}
Resumo
O presente estudo objetivou verificar os parâmetros psicométricos do Posttraumatic Growth Inventory - PTGI, apresentando evidências de validade fatorial, consistência interna e etapas da sua adaptação transcultural. A amostra foi composta por 205 participantes, sua maioria do sexo feminino (59,03\%), com idades variando entre 18 e 86 anos $(M=29,54 ; D P=11,43)$. Estes responderam ao PTGI, Life Stressor Checklist - Revised e perguntas demográficas. Nas análises fatoriais confirmatórias, observa-se o modelo pentaforial como mais adequado para o PTGI, sendo estatisticamente superior ao tri e ao unifatorial. A consistência interna de seus fatores (alfa de Cronbach) variou de 0,70 a 0,86 , com alfa geral de 0,92 , valores considerados aceitáveis. Conclui-se que essa medida, apresentando evidências de validade de construto, pode ser usada adequadamente em pesquisas no contexto em que foi adaptada, entretanto, recomenda-se o uso do instrumento em amostras mais heterogêneas e a realização de outras análises, observando sua invariância fatorial.

Palavras-chave: psicologia positiva, crescimento pós-traumático, posttraumatic growth inventory, validade, testes psicológicos
\end{abstract}

Posttraumatic Growth Inventory (PTGI): Adaptation and Factorial Validity in the Brazilian Northeast

\begin{abstract}
This study aimed to verify the psychometric parameters of the Posttraumatic Growth Inventory - PTGI, presenting evidence of factorial validity, internal consistency and steps of cross-cultural adaptation. The sample consisted of 205 participants from northeastern Brazilian cities, mostly female (59.03\%), aged between 18 and 86 years $(M=29.5$, SD $=11.4)$. Participants answered the PTGI, the Life Stressor Checklist - Revised, and demographic questions. The confirmatory factor analysis observed the penta-factorial model as more suitable for PTGI, being statistically superior to three and one-factor. The internal consistency of its factors (Cronbach's alpha) ranged from .70 to .86 , with general alpha of .92, values considered acceptable. It follows that this measurement, providing evidence of construct validity, can be properly used in research in the context in which it was adapted to, however, we recommend the use of the instrument in more heterogeneous samples and conducting other analyzes, observing its factorial invariance.

Keywords: positive psychology; post-traumatic growth; posttraumatic growth inventory; validity; psychological tests
\end{abstract}

Posttraumatic Growth Inventory (PTGI): Adaptación y Validez Factorial en el Noreste de Brasil

\section{Resumen}

Este estudio tuvo como objetivo verificar los parámetros psicométricos del Posttrumatic Growth Inventory - PTGI, presentando evidencias de validez factorial, consistencia interna y etapas de su adaptación transcultural. La muestra fue compuesta por 205 participantes, en su mayoría de sexo femenino (59,03\%), con edades entre 18 y 86 años $(M=29.5 ; S D=11.4)$. Ellos respondieron el PTGI, Life Stressor Checklist - Revised y preguntas demográficas. En los análisis factoriales confirmatorios se observa el modelo pentafactorial como el más adecuado para el PTGI, siendo estadísticamente superior al tri y al unifactorial.La consistencia interna de sus factores (alfa de Cronbach) varió de 0,70 a 0,86, con alfa general 0,92, valores considerados aceptables. Esta medida, por presentar evidencias de validez de constructo, puede ser utilizada adecuadamente en investigaciones en el contexto en que fue adaptada, sin embargo, se recomienda el uso del instrumento en muestras más heterogéneas y la realización de otros análisis, observando su invariancia factorial.

Palabras-clave: Psicología Positiva, crecimiento post-traumático; posttraumatic growth inventory; validez; tests psicológicos.

\section{Introdução}

No transcurso da vida, as pessoas estão suscetíveis a inúmeros acontecimentos adversos. Tais episódios (e.g., a vivência de um desastre natural, morte de uma pessoa próxima, vivenciar um acidente, entre outros) certamente despertam sentimento de tristeza, angústia e medo. Especificamente podem ocasionar problemas de natureza física, psíquica e social. Todavia, para algumas pessoas, tais acontecimentos passam a ser oportunidades de crescimento pessoal e relacional; para outras, são situações devastadoras que levam o indivíduo a significar como o fim de expectativas, de sonhos e até mesmo da vida (Calhoun \& Tedeschi, 2012). 
Autores, a exemplo de Maia e Resende (2008), afirmam que $80,5 \%$ das pessoas já vivenciaram mais do que um evento considerado traumático na vida. Essa assertiva é corroborada por Bonanno (2004), Ozer, Best, Lipsey e Weiss (2003), quando afirmam que a maioria das pessoas é exposta ao menos a uma situação violenta ou ameaçadora durante o seu ciclo de vida.

Alguns acontecimentos, raros ou não, são tomados como "potencialmente traumáticos" (Madeira, 2013), pois o significado do trauma é encarado de forma subjetiva, uma vez que nem todas as pessoas reagem de forma igual à situação e nem se desestabilizam em situações extremas. Assim, uma situação que, para algumas pessoas, é uma experiência estressora, pode não gerar consequências físicas ou psíquicas para outras.

Para além disso, a vivência de eventos estressores ou de uma experiência traumática pode servir como uma oportunidade para os indivíduos aprenderem e, portanto, crescerem enquanto pessoas (Bluvstein, Moravchik, Sheps, Schreiber, \& Bloch, 2012), fenômeno chamado de crescimento pós-traumático. $\mathrm{Na}$ linha desta compreensão, Ramos e Leal (2013) definem o crescimento pós-traumático (CPT) como um fenômeno caracterizado por mudanças psicológicas positivas experimentadas pelas pessoas que resulta de um processo de luta frente a vivência de eventos adversos. Sendo os eventos traumáticos oportunidades para crescer por meio da dor, abrir novos horizontes e reinterpretar as crenças (Lau et al., 2015). Evidenciando-se, assim, a crescente busca por temas afirmativos e construtivos, por meio de estudos que ampliam olhares e abordam os aspectos virtuosos e as forças pessoais (Cardoso, 2013; Noronha, Dellazzana-Zanon, \& Zanon, 2015).

As pesquisas sobre forças e virtudes foram fortemente impulsionadas pela Psicologia Positiva (Seligman \& Csikszentmihalyi, 2000). Apresentando-se como uma proposta de equilibrar o foco e os estudos produzidos na área da Psicologia (Reppold, Gurgel, \& Schiavon, 2015), pretende superar o enfoque na reparação de danos e focalizar no que faz a vida ter seu valor (Pacico \& Bastianello, 2014; Vázquez, 2013). Dentre os construtos estudados por essa abordagem, destaca-se o CPT. Este termo, crescimento, caracteriza o nível atingido pela pessoa que vai além do funcionamento anterior ao trauma, e pós-traumático faz referência ao crescimento que só acontece depois da vivência de um evento traumático, preparando e fortalecendo a pessoa para futuras situações (Fonseca, 2011).
Existem evidências de benefícios percebidos após experiências de vida desafiadoras documentadas desde os anos 1980 (Osei-Bonsu, Weaver, Eisen, \& Wal, 2012). Especificamente, nota-se que o interesse científico começou com estudos que relatavam mudanças positivas percebidas em diferentes situações, por exemplo, o quanto mulheres crescem quando sobrevivem a um estupro (Burt \& Katz, 1987), relatos de benefícios, diminuição de morbidades, em pacientes cardíacos após um primeiro ataque (Affleck, Tennen, Croog, \& Levine, 1987), mudanças de metas em universitários enlutados (Edmonds \& Hooker, 1992), além da identificação de menor impotência e maior resistência a eventos estressores em combates veteranos (Elder \& Clipp, 1989).

A partir disso, Tedeschi e Calhoun (1996) passam a estudá-las, caracterizando o crescimento pós-traumático, a fim de representar essas transformações psicológicas positivas depois da vivência traumática. Tais autores sistematizaram o construto em três categorias: (a) mudança de significado do selfou autopercepção; (b) mudança de significado das relações com os outros; e, por fim, (c) mudanças na filosofia de vida (Calhoun \& Tedeschi, 2012; Osei-Bonsu, et al., 2012; Tedeschi \& Calhoun, 1996).

A primeira categoria diz respeito à pessoa perceber-se que está mais forte e confiante em si mesma, desenvolvendo uma autoimagem de maior força e confiança para lidar com os futuros desafios, adversidades e situações que a pessoa julga que precisam ser mudadas (Tedeschi \& Calhoun, 1996). Além dessa percepção de maior força individual, está relacionada com o reconhecimento de mais recursos (Lindstrom, Cann, Calhoun, \& Tedeschi, 2013; Ramos \& Leal, 2013). Tal mudança é contraditória, sendo representada pela frase: "estou mais vulnerável, embora fortalecido(a)".

A segunda mudança mencionada, é nas relações interpessoais. As mais íntimas, como as expressivas, passam a ser mais intensas. Algumas pessoas comentam perceber mais conforto e confiança ao revelar seus pensamentos e emoções aos outros, entretanto, passam a selecionar com mais atenção as pessoas que o rodeiam. Outro componente importante é a sensação de maior compaixão por outros que passam por dificuldades, sendo mais característico frente àquelas semelhantes às suas (Calhoun \& Tedeschi, 2012; Fonseca, 2011).

A terceira categoria denominada de mudança na filosofia de vida ou, ainda, na espiritualidade é característica de pessoas que mudam de prioridades e opções em vários domínios de suas vidas (Lindstrom et al., 2013). Aumenta a apreciação do que se tem, as 
pequenas coisas e os detalhes são mais valorizados e distinguem-se entre o que é realmente importante e o que é secundário. Essa categoria também representa mudanças espirituais, existenciais e religiosas, aumento da fé e busca de significados de vida no processo de adaptação e crescimento (Calhoun \& Tedeschi, 2012; Tedeschi \& Calhoun, 1996).

Essas dimensões são as que originalmente foram organizadas para definir o construto crescimento pós-traumático (Tedeschi \& Calhoun, 1996), contudo, segundo investigações recentes (Duarte, 2012; Taku, Kilmer, Cann, Tedeschi, \& Calhoun, 2012), possivelmente influenciadas por estudos oriundos da Psicologia Positiva, esses domínios foram reformulados em cinco: (a) aumento da apreciação de vida no geral; (b) relações interpessoais mais significativas; (c) aumento do sentimento de força pessoal; (d) mudanças de prioridades; e (e) uma vida espiritual e existencial mais rica.

Dessa forma, atualmente, o CPT é caracterizado como um construto multidimensional, no qual os indivíduos podem experimentar mudanças positivas em algum desse domínio e negativas em outros (Ramos \& Leal, 2013). Destaca-se que, em muitos casos, sem a presença de emoções negativas, pelo menos no início, o crescimento não vai acontecer, haja vista a necessidade de coexistência de emoções individuais positivas e negativas para que esse fenômeno seja experimentado (Calhoun \& Tedeschi, 1999).

Essas mudanças não são raras de serem encontradas (Cho \& Park, 2013), a exemplo dos estudos com adolescentes com ideação suicida (Yu et al., 2010), sobreviventes de acidentes (Nishi, Matsuoka, \& Kim, 2010), adultos vítimas de assédio sexual (Ullman, 2014), estudantes universitários em luto (Duarte, 2012), pessoas que preenchem os critérios de avaliação de traumas segundo o DSM-IV (Osei-Bonsu et al., 2012), sobrevivente de doenças graves no coração (Bluvstein et al., 2012) e HIV/AIDS (Sawyer, Ayers, \& Field, 2010).

Tais estudos revelam a relevância do tema, especialmente na busca de compreensão (antecedentes e consequentes) no que diz respeito às aprendizagens obtidas pelas pessoas nesses contextos de sofrimento, as quais favoreceram, de alguma forma, o crescimento pessoal. No entanto, a produção cientifica nacional é escassa. Isso pode ser verificado em buscadores, como o PePSIC e SciElo Brasil, em dezembro de 2015. No caso, foi introduzido o termo "posttraumatic growth", sem estabelecer tempo, foram encontrados quatro artigos. Quando inserido o termo "crescimento pós-traumático" na busca, o total foi reduzido para apenas um registro, no entanto, nenhum descreve uma pesquisa com o crescimento pós-traumático como fenômeno observado. Portanto, apesar de crescente o interesse da Psicologia por essa temática, no cenário brasileiro, estudos ainda são insipientes.

Não obstante, considerando a relevância de conhecer mudanças psicológicas positivas após eventos traumáticos, julga-se importante lograr êxito em sua avaliação. Para esse fim, a literatura encontrada aponta que pelo menos sete instrumentos que mensuram alterações psicológicas positivas após eventos estressantes já foram construídos (Linley \& Joseph, 2004; Resende, Sendas, \& Maia, 2008), a saber: Stress-Related Growth Scale, Revised Stress - Related Growth Scale, Changes in Outlook Questionnaire, Thriving Scale, Illness Cognition Questionnaire, Perceived Benefit Scales, além do mais comumente utilizado e citado: o Posttraumatic Growth Inventory - PTGI. Objeto de interesse do presente estudo, trata-se de um instrumento desenvolvido por Tedeschi e Calhoun (1996), o qual apresentou índice de consistência interna total (alfa de Cronbach $=0,90$ ) e de suas cinco dimensões variando de 0,67 a 0,85 . Ademais, diferente dos outros instrumentos que mensuram mudanças positivas de maneira geral, o PTGI foi construído especificamente para mensurar o que se conceitua como crescimento pós-traumático, construto de interesse desta pesquisa.

Contudo, são encontrados resultados não conclusivos acerca da estrutura fatorial do PTGI. Não ficando claro se o crescimento pós-traumático é melhor representado por um ou mais fatores, logo, observa-se que, apesar do amplo uso dessa medida, a dimensionalidade do PTGI tem sido um tema de debate (Osei-Bonsu, et al., 2012). Haja vista os estudos com diversificadas estruturas fatoriais encontradas: com um fator (Joseph, Linley, \& Harris, 2005), dois fatores (Thege, Kovács, \& Balog, 2014), três fatores (Taku, Calhoun, Tedeschi, Gil-Rivas, \& Cann, 2007), quatro fatores (Ho et al., 2011), cinco fatores (Purc-Stephenson, 2014); e formas reduzidas da medida, com cinco fatores, representados por 15 itens (Liu et al., 2015) e 10 itens (Lamela, Figueiredo, Bastos, \& Martins, 2014), além da estrutura composta por seis fatores (Morris, Wilson, \& Chambers, 2013).

Evidencia-se o uso internacional dessa medida, com traduções em distintas línguas, como o japonês (Taku et al., 2007), italiano (Prati \& Pietrantoni, 2014), português de Portugal (Teixeira \& Pereira, 2013), holandês (Jaarsma, Pool, Sanderman, \& Ranchor, 2006), chinês (Lau et al., 2013), turco (Dirik \& Karanci, 2008) e hungáro (Thege et al., 2014). Ressalta-se que não foram encontrados estudos que apontassem 
para o uso do PTGI em português, do Brasil, o que demonstra uma lacuna que pode ser suprida por este estudo, justificando-o.

Logo, buscando contribuir com a produção científica acerca do construto, este estudo tem como objetivo apresentar a adaptação transcultural para o português brasileiro e verificar evidências de qualidades psicométricas do Posttrumatic Growth Inventory - PTGI em uma amostra do nordeste brasileiro, além de confrontar modelos estruturais alternativos.

\section{Método}

\section{Participantes}

Após dadas todas as instruções necessárias e ter o consentimento da participação do respondente, utilizou-se, como critério de inclusão na amostra, ter sofrido ou passado pelo menos por um dos acontecimentos estressantes ou traumáticos listados no Life Stressor Checklist (Maia \& Resende, 2008). Dessa forma, participaram deste estudo 205 pessoas de três cidades do nordeste brasileiro. Tratou-se de uma amostra por conveniência, ou seja, foram computadas as respostas dos participantes que aceitaram participar da pesquisa voluntariamente. Estas tinham idades variando entre 18 a 86 anos $(M=29,54, D P=11,43)$, sendo a maioria do sexo feminino $(59 \%)$ e solteira $(51,2 \%)$. Desse total, $36,7 \%$ das pessoas declararam possuir ensino superior incompleto como grau de escolaridade e com renda familiar média aproximada de $\mathrm{R} \$ 2.184,85$ (DP $=2.364,04)$.

\section{Instrumentos}

Life Stressor Checklist-Revised. Este instrumento foi traduzido para o português de Portugal por Maia e Resende (2008), a partir da Life Stressor Checklist - Revised (LSC - R., de Wolfe, Kimerling, Brow, Cheresman, \& Levin, 1996), nesta pesquisa, para a sua utilização, este foi adaptado para o português brasileiro. Destaca-se que este não foi utilizado como instrumento psicométrico (portanto, não havendo dados de validação), e sim como um critério de inclusão. Esse checklist consiste numa lista de 30 acontecimentos de vida adversos (e.g., morte inesperada de um familiar ou amigo próximo; ser assaltado ou sofrer um ataque físico). Um instrumento de autorrelato que os participantes marcam qual dos eventos que teriam acontecido em suas vidas e foram considerados como traumáticos, ou ainda, têm a possibilidade de apontarem outros que não constavam na lista original.
Posttraumatic Growth Inventory (PTGI). Foi desenvolvido por Tedeschi e Calhoun (1996) e avalia o grau de mudanças positivas percebidas pelo indivíduo após um acontecimento adverso. O PTGI original é uma escala de 21 itens e possui 5 fatores: Relação com os outros (itens 6, 8, 9, 15, 16, 20 e 21), Novas possibilidades (itens 3, 7, 11, 14 e 17), Mudança pessoal (itens 4, 10, 12 e 19), Mudança espiritual (itens 5 e 18) e Apreciação da vida (itens 1, 2 e 13), sendo que o valor total (somatório de todos os itens) corresponde ao índice de crescimento pós-traumático. Os itens, a exemplo do item 2: "Dou mais valor à minha vida" e do item 19: "Descobri que sou mais forte do que pensava", são respondidos com escala de respostas do tipo Likert que varia de 0 (Não experimentei mudança como resultado desse trauma) a 5 (Mudei completamente), em que consideram ter mudado em consequência de um determinado trauma. Evidenciase que esse instrumento não apresenta evidências de validade para a população brasileira.

Questões sociodemográficas. Conjunto de perguntas que objetivavam caracterizar a amostra, a exemplo de questões sobre a idade, o sexo, a renda e o estado civil.

\section{Procedimentos}

Inicialmente, buscou-se traduzir o PTGI para o português do Brasil, por meio do método do Back Translation (Sousa \& Rojjanasrirat, 2010), assim, a medida foi traduzida por dois psicólogos bilíngues e em seguida retraduzida para o inglês, com a colaboração de dois professores bilíngues de língua inglesa, que não conheciam o instrumento (tradução às cegas). Realizou-se esse método, a fim de averiguar se os itens das duas versões eram equivalentes, os tradutores estavam atentos às diferenças culturais de cada idioma, fazendo, quando necessário, as devidas adequações e adaptações na redação dos itens.

Posteriormente, após checar a correspondência dos itens originais e os traduzidos, submeteu-se a versão à próxima etapa de validação semântica, seguindo os procedimentos estabelecidos por Pasquali (2010). Uma amostra de 10 participantes integrantes da população-alvo do estudo, que foram distribuídos equitativamente entre homens e mulheres, verificou possíveis dificuldades de leitura e interpretação dos itens do instrumento utilizado. Na oportunidade, não foram apontados problemas para compreensão dos itens e orientações de como respondê-los, dessa forma, prosseguiu com o uso do instrumento. 
Desse modo, para a aplicação dos instrumentos, dois bolsistas de iniciação científica e dois colaboradores, devidamente treinados previamente, visitavam residências, escolhidas acidentalmente, em cidades do nordeste brasileiro. Na ocasião, convidavam as pessoas para participarem da pesquisa. Nesse momento, eram informados o objetivo geral do estudo, o caráter voluntário da participação, bem como garantido o anonimato das respostas e a possibilidade de desistir de sua participação no estudo a qualquer momento, sem nenhum ônus. Então, após aceitarem participar do estudo, os participantes eram solicitados a assinar o Termo de Consentimento Livre e Esclarecido. Os instrumentos eram respondidos de forma individual, sendo necessários, em média, 20 minutos para sua conclusão.

Destaca-se que foram asseguradas todas as recomendações éticas relacionadas às pesquisas com seres humanos, conforme o disposto na resolução 466/2012 do Conselho Nacional de Saúde, sendo a realização do estudo aprovado pelo Comitê de Ética em Pesquisa de uma Universidade localizada na região Nordeste (Parecer $\left.\mathrm{n}^{\circ} 886.238 / 2014\right)$.

\section{Análise de Dados}

Os softwares AMOS e IBM SPSS (ambos em suas versões 21) foram utilizados. Com o IBM SPSS, calculou-se estatísticas descritivas, com o fim de caracterização da amostra, além do alfa de Cronbach que serviu para avaliar a consistência interna da medida. Com o AMOS foram realizadas análises fatoriais confirmatórias (AFCs), testando a adequação do modelo de cinco fatores, além de compará-lo a soluções alternativas. Esse tipo de análise oferece alguns indicadores de ajuste do modelo teórico aos dados empíricos, sendo os seguintes amplamente considerados (Byrne, 2010; Tabachnick \& Fidell, 2013):

(a) $\chi^{2} / g l$ (razão entre o $\chi^{2}$ e o número de graus de liberdade) é uma tentativa de tornar o $\chi^{2}$ menos dependente do tamanho amostral. Valores entre 2 e 3 indicam um bom ajuste. (b) Comparative Fit Index (CFI) é um índice comparativo. Frequentemente, valores a partir de 0,90 são referências de um modelo ajustado. (c) Tucker-Lewis Index (TLI). Apresenta uma medida de parcimônia entre os índices do modelo proposto e do modelo nulo. Varia de zero a um, com índice aceitável acima de 0,90. (d) Root-Mean-Square Error of Approximation (RMSEA) e seu intervalo de confiança de $90 \%$ (IC 90\%). Recomendando-se valores entre 0,05 e 0,08, admitindo-se até 0,10 .
Ademais, com o fim de comparar os modelos alternativos, consideraram-se os seguintes: (1) o Consistent Akaike Information Criterion (CAIC), (2) o Expected Cross-Validation Index (ECVI) e (3) a diferença entre os qui-quadrados e os respectivos graus de liberdade dos modelos $\left(\Delta \chi^{2}\right)$.

\section{Resultados}

Buscando reunir evidências psicométricas sobre o PTGI, inicialmente decidiu-se verificar a adequação do modelo original, pentafatorial, encontrado no estudo de Tedeschi e Calhoun (1996). Para tanto, realizou-se uma análise fatorial confirmatória (AFC), tendo como matriz de entrada a de variância-covariância, adotando o estimador ML (Maximum Likelihood). De acordo com os resultados da SEM (Structural Equation Modeling), os indicadores de adequação de ajuste desse modelo ficaram próximo do recomendado: $\chi^{2}(179)=357,71, p<$ 0,$001 ; \chi^{2} / g l=2,00, \mathrm{CFI}=0,91, \mathrm{TLI}=0,89$, RMSEA $=$ $0,07(\mathrm{IC} 90 \%=0,06-0,08)$.

Nesse sentido, procurando identificar um modelo mais adequado, foram observados os IMs (Índices de Modificação) para as saturações (lambdas, $\lambda$ ) e os erros de medida (deltas, $\delta$ ). Tais índices, refletem uma aproximação de quanto o qui-quadrado pode diminuir quando adicionado alguma relação é adicionada, dessa forma vão gradualmente apresentando melhores ajustes do modelo aos dados (Byrne, 2010). Não obstante, destaca-se que buscou apoiar-se no princípio da parcimônia estabelecendo o mínimo possível de relações entre erros, no caso duas. Concretamente, constatou-se que seria recomendável $(\mathrm{IM}=10,83)$ correlacionar os $\delta$ dos itens 3 (Tenho novos interesses) e 7 (Estabeleci um novo rumo para minha vida) do fator Novas Possibilidades, além dos $\delta$ dos itens 1 (Mudei as minhas prioridades acerca do que é importante na vida) e 2 (Dou mais valor à minha vida) do fator Apreciação da Vida (IM = 20,04). Procedendo dessa maneira, a estrutura fatorial resultante reuniu melhores indicadores de ajuste aos dados empíricos: $\chi^{2}(177)=324,93, p<0,001$, $\chi^{2} / g l=1,84$, CFI $=0,92$, TLI $=0,91$, RMSEA $=0,06$ (IC90\% $=0,05-0,07)$. É possível observar o modelo testado na Figura 1, ressalta-se que todas as saturações foram estatisticamente diferentes de zero $(\lambda \neq 0 ; z>$ $1,96, p<0,05)$.

A média de pontuação total do PTGI foi 74,28 $(D P=18,09)$. As estatísticas descritivas de cada escala foram: Fator 1. Relação com os outros, $M=23,18$ $(D P=7,64)$; Fator 2. Novas possibilidades, $M=$ 


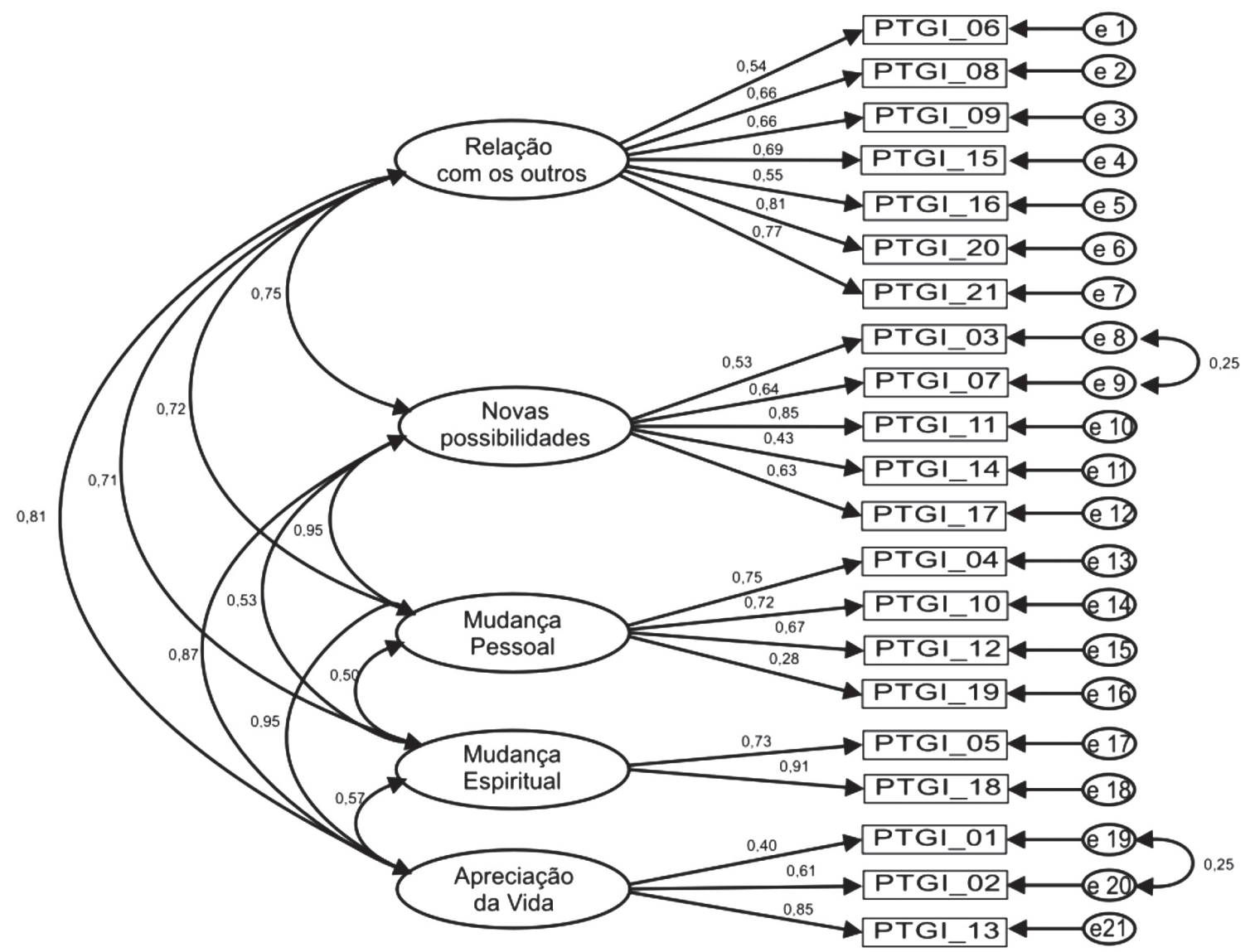

Figura 1. Modelo pentafatorial do fenômeno CPT.

18,03 $(D P=4,66)$; Fator 3. Mudança pessoal, $M=$ $15,40(D P=3,73)$; Fator 4. Mudança espiritual, $M$ $=6,49(D P=3,41)$; Fator 5. Apreciação da vida, $M$ $=11,17(D P=3,15)$. Calculou-se, ainda, para cada fator, sua consistência interna (alfa de Cronbach, $\alpha$ ) e homogeneidade (correlação média inter-itens $/ r_{\mathrm{i} . \mathrm{i}}$ ), apontando os seguintes resultados: Fator 1. Relação com os outros, $\alpha=0,86, r_{\mathrm{i} . \mathrm{i}}=0,46$; Fator 2. Novas possibilidades, $\alpha=0,77, r_{\mathrm{ij}}=0,41$; Fator 3. Mudança pessoal, $\alpha=0,70, r_{\mathrm{i} . \mathrm{i}}=0,37$; Fator 4. Mudança espiritual, $\alpha=0,79, r_{\mathrm{ii}}=0,66$; Fator 5 . Apreciação da vida, $\alpha=0,70, r_{\mathrm{i.i}}=0,44$ e por fim, Fator geral. PTGI, $=$ $0,92, r_{\mathrm{i} . \mathrm{i}}=0,36$.

Diante do pouco consenso acerca da estrutura do PTGI, decidiu-se verificar a pertinência do modelo originalmente proposto, com cinco fatores, quando comparado a modelos alternativos. Dessa forma, o modelo pentafatorial foi contrastado com modelos alternativos mais encontrados na literatura: (1) modelo trifatorial, levado em conta os temas centrais do crescimento pós-traumático na estruturação do fenômeno, os itens foram arranjados na estrutura teórica esperada, representada por Fator 1: união dos itens que compõe os fatores 1 e 2 do PTGI original, Fator 2: itens dos fatores 3 e 5, e Fator 3: itens do Fator 4 (Calhoun \& Tedeschi, 2012; Tedeschi \& Calhoun, 1996); (2) modelo unifatorial, considerando o crescimento pós-traumático como um construto unifatorial, apresentando todos os itens saturando em uma única dimensão (Joseph et al., 2005; Osei-Bonsu et al., 2012). Na Tabela 1, são expostos os índices de bondade de ajuste para cada modelo testado.

De acordo com a Tabela 1, o modelo 1 com cinco fatores apresentam os resultados mais promissores, o qual descreve a estrutura original do PTGI (Relação com os outros, Novas possibilidades, Força pessoal, Mudança espiritual e Apreciação da vida), mostrando-se o mais adequado (e.g., menores CAIC, ECVI e $\chi^{2}$ ), sendo estatisticamente superior ao modelo com três fatores, que, por sua vez, mostrou-se mais adequado 
Tabela 1

Comparação de Três Modelos do PTGI

\begin{tabular}{lcccccccc}
\hline Modelos & $\chi^{2}$ & Gl & $\chi^{2} / g l$ & CFI & TLI & RMSEA (IC90\%) & CAIC & ECVI \\
\hline cinco & 357,71 & 179 & 2,00 & 0,91 & 0,89 & $0,07(0,06-0,08)$ & 686,51 & 2,26 \\
três & 483,83 & 186 & 2,60 & 0,84 & 0,82 & $0,09(0,08-0,10)$ & 768,37 & 2,81 \\
um & 586,95 & 189 & 3,10 & 0,79 & 0,77 & $0,10(0,09-0,11)$ & 852,51 & 3,29 \\
\hline
\end{tabular}

Nota. $n=205$. Modelos: pentafatorial (original), trifatorial (modelo teórico proposto) e unifatorial (todos os 21 itens saturando em um único fator). $\chi^{2} / g l=$ razão qui-quadrado/graus de liberdade; CFI = Comparative Fit Index; TLI = Tucker-Lewis Index; RMSEA = Root-Mean-Square Error Aproximation, IC 90\% = Intervalo de Confiança de 90\%; CAIC = Consistent Akaike Information Criterion; ECVI = Expected Cross-Validation Index.

que o modelo unifatorial. Entretanto, com o fim de obter uma prova de significância da adequabilidade do modelo pentafatorial, comparou-se seu qui-quadrado com os dos modelos trifatorial $\left[\Delta \chi^{2}(7)=126,13, p<\right.$ $0,001]$ e unifatorial $\left[\Delta \chi^{2}(10)=229,24, p<0,001\right]$.

Dado o exposto, resume-se que foram reunidas evidências de validade fatorial e consistência interna do Posttraumatic Growth Inventory numa amostra do nordeste brasileiro. Ficando evidente que o modelo pentafatorial é o mais adequado para representar o fenômeno crescimento pós-traumático da maneira que foi operacionalizado, sendo claramente melhor do que aqueles outros dois modelos alternativos apresentados, o trifatorial e o unifatorial.

\section{Discussão}

O presente estudo teve como objetivo conhecer evidências de validade fatorial e consistência interna do PTGI, além de testar modelos fatoriais alternativos dessa medida, confia-se que os objetivos tenham sido cumpridos. A seguir são discutidos, a luz da literatura consultada, os principais resultados aqui apresentados.

O Posttraumatic Growth Inventory trata-se de uma medida psicométrica desenvolvida por Tedeschi e Calhoun (1996) para avaliar a mudança pessoal positiva após passar por eventos adversos. Assim como no estudo original, a versão final, em português do Brasil, do PTGI ficou composto por 21 itens distribuídos estruturalmente em cinco dimensões: (1) Relação com os outros; (2) Novas possibilidades; (3) Força pessoal; (4) Mudança espiritual; e (5) Apreciação da vida. Essa estrutura e número de itens converge com resultados recentes da literatura (Taku et al., 2012), inclusive mostrando-se invariante para amostras com doenças crônicas (reumatóide e doença inflamatória intestinal; Purc-Stephenson, 2014).
A estrutura pentafatorial quando contrastada com outros dois modelos, unifatorial, em que todos os itens saturaram em um único fator, representando, todos eles, a dimensão Crescimento pós-traumático, e o segundo modelo, trifatorial, representando as três grandes categorias propostas por Tedeschi e Calhoun (1996) na estruturação teórica do crescimento pós-traumático, revelou-se mais adequado, considerando indicadores de ajustes comumente tidos em conta para comparar modelos alternativos (CAIC, ECVI e $\Delta \chi^{2}$; Byrne,2010; Tabachnick \& Fidell, 2013).

Dessa forma, o PTGI, para a amostra aqui utilizada, é claramente melhor representado como composto por cinco dimensões, versão mais atual da sistematização desse fenômeno (Taku et al., 2012), reunindo evidências da nova redefinição da quantidade dos domínios do construto, que inicialmente eram três (Tedeschi, \& Calhoun, 1996).

A multidimensionalidade do CPT é defendida, pois, com maior número de dimensões, há uma aproximação mais real com o fenômeno. Permitindo assim avaliar e, consequentemente, observar, nos indivíduos avaliados, crescimento em alguma(s) dimensão(ões) e não em outras, o que caracterizaria o enfretamento individual do trauma pelos indivíduos (Ramos \& Leal, 2013).

Ademais, segundo Lau et al., (2013) o modelo pentafatorial do PTGI, encontrado por Tedeschi e Calhoun (1996), vem se demonstrando adequado psicometricamente para avaliação do crescimento pós-traumático, apesar de, recentemente, estudos sugerirem diferenças culturais existentes na mensuração de crescimento pós-traumático (Splevins, Cohen, Bowley, \& Joseph, 2010; Taku et al., 2012). Inclusive com as versões com menor quantidade de itens, de 15 itens (Liu et al., 2015) e 10 itens (Lamela et al., 2014).

No que diz respeito a precisão do PTGI, avaliada a partir de índices de consistência interna (alfa 
de Cronbach; Nunnally, 1978), apresentando alfas de Cronbach iguais ou superiores a 0,70 é considerada adequada (Blunch, 2008; Cohen, Swerdlik, \& Sturman, 2014), além de possuir índice de homogeneidade meritório (Clark \& Watson, 1995). Apesar de satisfatórias, as evidências de fidedignidade, cabe ponderar que o número de itens da maior parte dos fatores é pequeno, variando entre dois e quatro itens, o que certamente afeta os índices estarem próximos aos valores limites recomendados (Oviedo \& Campo-Arias, 2005).

Sabendo da importância de se contar com instrumentos que apresentem qualidades psicométricas adequadas e fundamentação teórica sólida que justifique seu uso e sua utilidade para se compreender seus antecedentes e consequentes (Clark \& Watson, 1995), acredita-se que o presente estudo apresente uma valiosa contribuição. Nele é apresentado um instrumento com suas qualidades asseguradas, que se torna alternativa, em português brasileiro, para pesquisadores interessados em avaliar e conhecer os correlatos do crescimento pós-traumático.

Ademais, observa-se que os estudos acerca do fenômeno crescimento pós-traumático e as mudanças positivas frente à vivência do trauma têm ganhado, cada vez mais, reconhecimento pela comunidade científica, sobretudo a Psicologia, que durante muitos anos, ignorou a perspectiva positiva, enfatizando apenas o alívio de sintomas e correções dos danos (Reppold et al., 2015). No entanto, o estudo sobre os fatores que predizem ou que resultam desse processo ainda são inconclusivos e contraditórios (Lindstrom et al., 2013), o que reforça a importância de se contar com medidas psicometricamente ajustadas.

Apesar de terem sido alcançados os objetivos propostos, este, a exemplo de qualquer outro estudo científico, não está isento de limitações. Nesse sentido, destaca-se o viés amostral. Por se tratar de uma amostra acidental (por conveniência; Cozby, 2003; Tabachnick \& Fidell, 2013). Implica em o leitor avaliar os resultados ora apresentados, com ressalvas, não os extrapolando para além da amostra aqui utilizada. Outra importante limitação do estudo é a ausência de uma avaliação das evidências externas do PTGI, pois são proporcionadas evidências de validade interna, no entanto, carece de evidências de validade com base na relação com outras variáveis.

Contudo, ressalta-se que aqui não se pretendeu generalizar os resultados, mas averiguar evidências de validade e precisão do PTGI no contexto estudado. Sugere-se que estudos futuros utilizem amostras maiores e mais heterogêneas, de preferência representativas da população de interesse. Além de testar outros aspectos da medida, a exemplo de sua validade convergente, discriminante e a invariância fatorial. Dessa maneira, será possível encorpar as evidências aqui apresentadas acerca das propriedades psicométrica do PTGI, dando maior segurança para os pesquisadores que optarem pelo seu uso.

\section{Referências}

Affleck, G., Tennen, H., Croog, S., \& Levine, S. (1987). Causal attributions, perceived benefits, and morbidity after a heart attack: An 8-year study. Journal of Consulting and Clinical Psychology, 55(1), 29-35. doi: 10.1037/0022-006X.55.1.29

Bonanno, G. A. (2004). Loss, trauma, and human resilience: Have we underestimated the human capacity to thrive after extremely aversive events? American Psychologist, 59, 20-28. doi: 10.1037/0003-066X.59.1.20

Blunch, N. (2008). Introduction to structural equation modelling using SPSS and AMOS. London: Sage Publications.

Bluvstein, I., Moravchick, L., Sheps, D., Schreiber S., \& Bloch, M. (2012). Posttraumatic growth, posttraumatic stress symptoms and mental health among coronary heart disease survivors. J Clin Psychol Med Settings, 20, 164-172. doi: 10.1007/ s10880-012-9318-z.

Burt, M. R., \& Katz, B. L. (1987). Dimensions of recovery from rape: Focus on growth outcomes. Journal of Interpersonal Violence, 2(1), 57-81. doi: 10.1177/088626087002001004

Byrne, B. M. (2010). Structural equation modeling with Amos: Basic concepts, applications, and programmimg (2 ed.). New York, NY: Routledge.

Calhoun, L. G., \& Tedeschi, R. G. (1999). Facilitating posttraumatic growth: A clinician's guide. Mabwah, NJ: Lawrence Erlbaum.

Calhoun, L. G., \& Tedeschi, R. G. (2012) Crescimento pós-traumático em intervenções clínicas cognitivocomportamentis. Em Caballo, V. E., Manual para tratamento cognitivo-comportamental dos transtornos psicológicos da atualidade: Intervenções em crise, transtornos da personalidade e do relacionamento e psicologia da saúde. São Paulo: Santos.

Psico-USF, Bragança Paulista, v. 22, n. 3, p. 449-460, set./ dez. 2017 
Cardoso, T., (2013). Construção e validação de uma escala dos atributos pessoais da resiliência (Dissertação de mestrado). Universidade Metodista de São Paulo, Faculdade de Saúde. São Bernardo do Campo.

Cho, D., \& Park, C. L. (2013). Growth following trauma: Overview and current status crecimiento tras el trauma: Revisión general y estado actual. Terapia psicológica, 31(1), 69-79. Recuperado de http:// www.scielo.cl/pdf/terpsicol/v31n1/art07.pdf

Clark, L. A., \& Watson, D. (1995). Constructing validity: Basic issues in objective scale development. Psychological Assessment, 7(3), 309-319. Recuperado de http://www.personal.kent.edu/ dfresco/ CRM_Readings/Clark_and_Watson_1995.pdf

Cohen, R. J., Swerdlik, M. E., \& Sturman, E. D. (2014). Testagem e avaliação psicológica: Introdução a testes e medidas. ( $8^{\circ}$ ed) São Paulo: AMGH.

Cozby, P. C. (2003). Métodos de pesquisa em ciências do comportamento. São Paulo: Atlas

Dirik, G., \& Karanci, A., N. (2008). Variables related to posttraumatic growth in turkish rheumatoid arthritis patients. J Clin Psychol Med Settings, 15(3), 93-203. doi: 10.1007/s10880-008-9115-x

Duarte, A. M. P. (2012). Experiência do luto e crescimento pós-traumático à luz da perspectiva da vinculação numa amostra de estudantes universitários (Dissertação de mestrado). Centro Regional de Braga, Faculdade de Filosofia.

Edmonds, S., \& Hooker, K. (1992). Perceived changes in life meaning following bereavement. Omega: Journal of Death and Dying, 25(4), 307-318. doi: 10.2190/ te $7 \mathrm{q}-5 \mathrm{~g} 45$-bety-x $1 \mathrm{tt}$

Elder, G. H., \& Clipp, E. C. (1989) Combat experience and emotional health: Impairment and resilience in later life. Journal of Personality, 57(2), 311-341. doi: 10.1111/j.1467-6494.1989.tb00485.x.

Fonseca, C. C. (2011). Crescimento pós-traumático: O impacto de falar sobre os aspectos positivos de uma experiência traumática (Dissertação de mestrado). Universidade de Lisboa, Faculdade de Psicologia.

Jaarsma, T. A., Pool, G., Sanderman, R., \& Ranchor, A. V. (2006). Psychometric properties ofthe Dutch version of the Posttraumatic Growth Inventory among cancer patients. Psycho-Oncology, 15(10), 911920. doi: $10.1002 /$ pon.1026
Joseph, S., Linley, P. A., \&Harris, G. J. (2005). Understanding positive change following trauma and adversity: Structural clarification. Journal of Loss and Trauma, 10(1) 83-96. doi: 10.1080/15325020490890741.

Lamela D., Figueiredo B., Bastos A., \& Martins H. (2014). Psychometric properties of the Portuguese version of the posttraumatic growth inventory short form among divorced adults. European Journal of Psychological Assessment. 30(1):3-14. doi: 10.1027/1015-5759/a000161.

Lau, J. T. F., Yeung, N. C. Y., Yu, X., Zhang, J., Mak, W. W. S., Lui, W. W. S., \& Zhang, J. (2013). Psychometric Properties of the Chinese Version of the Revised Posttraumatic Growth Inventory for Children (PTGI-C-R). Asia-Pacific Journal of Public Health, 27(2), 310-320. doi: 10.1177/1010539513479967

Lindstrom, C. M., Cann, A., Calhoun, L. G., \& Tedeschi, R. G. (2013). The relationship of core belief challenge, rumination, disclosure, and sociocultural elements to posttraumatic growth. Psychological Trauma, 5(1), 50-55. doi: 10.1037/a0022030

Linley, P. A., \& Joseph, S. (2004). Positive change following trauma and adversity: A review. Journal of Traumatic Stress, 17(1), 11-21. doi: 10.1023/B:JOTS $.0000014671 .27856 .7 \mathrm{e}$

Liu, J. E., Wang, H. J., Hua, L., Chen, J., Wang, M. L., \& Li. Y. Y. (2015). Psychometric evaluation of the simplified chinese version of the posttraumatic growth Inventory for assessing breast cancer survivors. European Journay of Oncology Nursing. 19(4), 391-396. doi: 10.1016/j.ejon.2015.01.002

Madeira, S. R. M. (2013). Relação entre as características da situação vivida, suporte social e resiliência, após a vivência de um acontecimento potencialmente traumático (Dissertação de mestrado). Universidade de Lisboa, Faculdade de psicologia.

Maia, A., \& Resende, C. (2008). Dados de exposição potencialmente traumática na população Portuguesa utilizando as Versão Portuguesas do Life Events Checklist e o Life Stressor Checklist - Revised. Braga: Psiquilibrios Edições.

Morris, B. A., Wilson, B., \& Chambers, S. K. (2013). Newfound compassion after prostate cancer: A psychometric evaluation of additional items in the Posttraumatic Growth Inventory. Supportive Care in Cancer, 21(12), 3371-3378. doi: 10.1007/ s00520-013-1903-7 
Oviedo, H. C., \& Campo-Árias, A. (2005). Aproximación al uso del coeficiente alfa de Cronbach. Revista Colombiana de Psiquiatría, 34(4), 527-580. Recuperado de http://www.redalyc. org/pdf/806/80634409.pdf

Osei-Bonsu, P. E., Weaver, T. L., Eisen, S. V., \& Wal, J. S. V. (2012). Posttraumatic growth inventory: Factor structure in the context of DSM-IV traumatic events. International Scholarly Research Network, 2012(2012). doi: 10.5402/2012/937582

Ozer, E., Best, S., Lipsey, T., \& Weiss, D. (2003). Predictors of posttraumatic stress disorder an symptoms in adults: A meta-analysis. Psychological Bulletin, 129(1), 52-79. Recuperado de http://www.ncbi. nlm.nih.gov/pubmed/12555794

Nishi, D., Matsuoka, Y., \& Kim, Y. (2010). Posttraumatic growth, posttraumatic stress disorder and resilience of motor vehicle accident survivors. BioPsychoSocial Medicine, 4(7). doi: 45678905678904567890567890

Noronha, A. P. P., Dellazzana-Zanon L. L., \& C. Zanon. (2015) Internal structure of the characters strengths scale in Brazil. Psico-USF, 20(2), 229-235. doi: 10.1590/1413-82712015200204

Nunnally, J. C. (1978). Psychometric theory. New York: McGraw-Hill Inc.

Pacico, J. C., \& Bastianello, M. R. (2014). As origens da psicologia positiva e os primeiros estudos brasileiros. In Hutz, C. S. Avaliação em Psicologia Positiva. Porto Alegre: Artes Médicas.

Pasquali, L. (2010). Instrumentação psicológica: Fundamentos e práticas. Porto Alegre, RS: Artmed.

Prati G., \& Pietrantoni L. (2014). Italian adaptation and confirmatory analysis on the full and the short form of the posttraumatic growth inventory. Journal of Loss and Trauma.19(1):12-22. doi: 10.1080/15325024.2012.734203

Purc-Stephenson, R. J. (2014). The posttraumatic growth inventory: Factor structure and invariance among persons with chronic diseases. Rehabilitation Psychology, 59(1), 10-18. doi: 10.1037/a0035353

Ramos, C., \& Leal, I., (2013). Posttraumatic growth in the aftermath of trauma: A literature review about related factors and application contexts. Psychology, Community \& Health, 2(1), 43-54. doi:10.5964/pch. v2i1.39
Reppold, C. T., Gurgel, L. L., \& Schiavon, C., C. (2015). Research in positive psychology: A systematic literature review. Psico-USF, Bragança Paulista, 20(2) 275-285. doi: 10.1590/1413-82712015200208

Resende, C., Sendas, S., \& Maia, A. (2008). Estudo das características psicométricas do Posttraumatic Growth Inventory - PTGI - (Inventário de Crescimento Pós-Traumático) para a população portuguesa. Em A. P. Noronha, C. Machado, L. Almeida, M. Gonçalves, S. Martind \& V. Ramalho (Ed.), Actas da XIII Conferência Internacional de Avaliação Psicológica: Formas e Contextos (pp. 1-16). Braga: Psiquilibrios Edições.

Sawyer, A., Ayers, S., \& Field, A. P. (2010). Posttraumatic growth and adjustment among individuals with cancer or HIV/AIDS: A meta-analysis. Clinical Psychology Review, 30, 436-447. doi: 10.1016/j. cpr.2010.02.004

Seligman, M. E. P., \& Csikszentmihalyi, M. (2000). Positive psychology: An introduction. American Psychologist,55(1), 5-14. doi:10.1037/0003-066X.55.1.5.

Sousa, V., \& Rojjanasrirat, W. (2010). Translation, adaptation and validation of instruments or scales for use in cross-cultural health care research: A clear and user friendly guideline. Journal of Evaluation in Clinical Practice, 17(2), 268-274. doi: 10.1111/j.1365-2753.2010.01434.x

Splevins, K., Cohen, K., Bowley, J., \& Joseph, S. (2010). Theories of posttraumatic growth: Cross-cultural perspectives. Journal of Loss and Trauma, 15(3), 259277. doi: 10.1080/15325020903382111

Tabachnick, B. G., \& Fidell, L. S. (2013). Using multivariate statistics ( $6^{\mathrm{a}}$ ed.). Nova Iorque: Allyn \& Bacon.

Taku, K., Calhoun, L. G., Tedeschi, R. G., Gil-Rivas, V., \& Cann, A. (2007). Examining posttraumatic growth among Japanese university students. Journal of Japanese Clinical Psychology, 20(4), 353-367. doi: 10.1080/10615800701295007

Taku, K., Kilmer, R. P., Cann, A., Tedeschi, R. G., \& Calhoun, L. G. (2012) Exploring posttraumatic Growth in Japanese Youth. Psychological Trauma: Theory, Research, Practice, and Policy, 4(4), 411-419. doi: $10.1037 /$ a0024363

Tedeschi, R. G., \& Calhoun, L. G. (1996). The Posttraumatic growth inventory: Measuring the positive 
legacy of Trauma. Journal of Traumatic Stress, 9(3), 455-471. Recuperado de http://www.ncbi.nlm. nih.gov/pubmed/8827649

Teixeira, R. J., \& Pereira, M. G. (2013). Growth and the cancer caregiving experience: Psychometric properties of the Portuguese Posttraumatic Growth Inventory. Families, Systems \& Health, 31(4), 382395. doi: $10.1037 /$ a0032004

Thege, B., K., Kovács, E., \& Balog, P. (2014) A bifactor model of the posttraumatic growth inventory. Health Psychology \& Behavioural Medicine, 2(1), 529540. doi: 10.1080/21642850.2014.905208

Ullman, S. E. (2014). Correlates of posttraumatic growth in adult sexual assault victims. Traumatology, 20(3) 219-224. doi: 10.1037/h0099402

Vázquez, C. (2013). Positive psychology and its enemies: A reply based on scientific evidence. Papeles del Psicólogo, 34, 3-25. Recuperado de https://www.
researchgate.net/publication/259257461_Positive_psychology_and_its_enemies_A_reply_based_ on_scientific_evidence

Wolfe, J., Kimerling, R. Brown, P. Chresman K., \& Levin, K. (1996). Psychometric review of the life stressor checklist-revised Em B. Stamm, (Ed.), Instrumentation in stress, trauma, and adaptation, (pp. 144-151), Sidran Press, Lutherville, MD.

Yu, X., Lau, J. T. F., Zhang, J., Mak, W. W. S., Choi, K. C., Lui, W. W. S., Zhang, J., \& Chan, E. Y. Y. (2010). Posttraumatic growth and reduced suicidal ideation among adolescents $\mathrm{t}$ month 1 after the 5.12.2008 Sichuan Earthquake. Journal of Affective Disorders. 123. 327-331. doi: 10.1016/j.jad.2009.09.019.

Recebido em: 18/02/2016

Reformulado em: 08/05/2016; 20/06/2016

Aprovado em: 22/06/2016 
Sobre os autores:

Emerson Diógenes de Medeiros é psicólogo, mestre e doutor em Psicologia Social pela UFPB. Atualmente é professor adjunto do curso de graduação em Psicologia da Universidade Federal do Piauí (Campus de Parnaíba) e coordena o Laboratório de Avaliação Psicológica do Delta - LABAP. Seus interesses de pesquisa centram-se em Psicologia Social (valores humanos e atitudes) e construção e adaptação de medidas psicológicas.

E-mail: emersondiogenes@gmail.com

Ricardo Neves Couto possui formação em Psicologia pela Universidade Federal do Piauí e atualmente é mestre e doutorando em Psicologia Social pelo Departamento de Psicologia da UFPB. É membro do Núcleo de Estudos em Desenvolvimento Humano, Educacional e Social (NEDHES) e tem interesse por pesquisas na área da Psicologia Social (valores humanos e atitudes), Psicologia Positiva, além de construção e adaptação de medidas psicológicas.

E-mail: r.nevescouto@gmail.com

Patrícia Nunes da Fonsêca possui formação e licenciatura em Psicologia, mestrado e doutorado em Psicologia Social pela Universidade Federal da Paraíba. É docente da Universidade Federal da Paraíba do Departamento de Psicopedagogia e Pós-Graduação em Psicologia Social, coordenadora do Núcleo de Estudos em Desenvolvimento Humano, Educacional e Social (NEDHES) e tem interesse por pesquisas nas áreas social, escolar e do desenvolvimento humano.

E-mail:pnfonseca.ufb@gmail.com

Paulo Gregório Nascimento da Silva é psicólogo pela Universidade Federal do Piauí (2014), mestre em Psicologia (ênfase em processos psicossociais) pelo Colegiado de Psicologia da Universidade Federal do Vale do São Francisco, Petrolina, Pernambuco, integrante do Laboratório de Avaliação Psicológica do Delta - LABAP. Interessa-se por pesquisas na área da Psicologia Social (valores humanos), Psicologia Positiva, construção e adaptação de medidas psicológicas.

E-mail:silvapgn@gmail.com

Paloma Cavalcante Bezerra de Medeiros é graduada em Psicologia nas habilitações licenciatura e formação de psicólogo pela Universidade Federal da Paraíba (UFPB), doutora em Psicologia Social pelo programa de Pós-Graduação em Psicologia Social da UFPB, atualmente é professora assistente do Departamento de Psicologia da Universidade Federal do Piauí. Sua principal experiência é na área de Neurociência Social.

E-mail:palomacbmedeiros@gmail.com

Contato com os autores:

Prof. Emerson Diógenes de Medeiros

Postal: Universidade Federal do Piauí

Departamento de Psicologia

Laboratório de Avaliação Psicológica do Delta - LABAP

Av. São Sebastião, 2819

Parnaíba-PI, Brasil

CEP: 64202-020 